\title{
Optimization of Concentric Ring Array Geometry for 3D Beam Scanning
}

\author{
Li Zhang, Yong-Chang Jiao, and Bo Chen \\ National Key Laboratory of Antennas and Microwave Technology, Xidian University, P.O. Box 377, Shaanxi, Xi'an 710071, China
}

Correspondence should be addressed to Li Zhang, lizhang@mail.xidian.edu.cn

Received 16 January 2012; Revised 29 February 2012; Accepted 3 March 2012

Academic Editor: Zhongxiang Q. Shen

Copyright () 2012 Li Zhang et al. This is an open access article distributed under the Creative Commons Attribution License, which permits unrestricted use, distribution, and reproduction in any medium, provided the original work is properly cited.

Optimization of the element placement in a concentric ring array for three-dimensional (3D) beam scanning with minimum peak sidelobe levels (SLLs) is addressed in this paper. In order to achieve 3D beam scanning with the lowest peak SLL, both the radius and the element spacing of each ring are optimized. Moreover, the aperture size is a very important constraint for the array, since there is an upper limit for the aperture size of a given array in real-life environment. Hence, in our optimization design, the maximum radius of the concentric ring array is constrained. Through the optimization, the peak SLL of the optimal concentric ring array is about $6 \mathrm{~dB}$ lower than that of the uniform concentric ring array, but the directivity is reduced by only $0.3 \mathrm{~dB}$.

\section{Introduction}

Antenna arrays for radar tracking, remote sensing, biomedical imaging, satellite and ground communications have often to support three-dimensional (3D) scanning with a suitable beampattern shape in the given angular region [1]. Towards this end, planar arrays have to be used and large apertures are necessary to provide satisfactory angular resolutions along both azimuth and elevation [1]. Among planar arrays, circular array has received considerable interest. Circular array can consist of either a single ring $[2,3]$ or multiple concentric rings [4-10]. However, uniformly excited and equally spaced circular ring array has high directivity but it usually suffers from high side lobe level (SLL). Therefore, to gain high performance of the circular array, the geometry and weights can be optimized. In [2], the weights and positions were simultaneously optimized in order to reduce the SLL in the azimuthal plane. In [3], three different evolutionary algorithms were employed to optimize the amplitude and phase excitations of the circular array for designing the beam scanning with the lowest peak SLL in the whole azimuthal plane. Amplitude weights for a concentric ring array can be found to yield an array factor that is invariant pattern over a specified bandwidth [4]. Kumar and Branner [5] proposed a method of lowering sidelobe level through optimizing the ring radii. In [6], an artificial neural network (ANN) with hidden Bessel activation functions was employed to design ultralow sidelobe level concentric ring arrays. In [7], both the ring radii and the number of elements in each ring were optimized to achieve the lowest peak SLL. Pathak et al. [8] synthesized the thinned concentric ring array to suppress the SLL at boresight. The weights and geometry were simultaneously optimized in order to determine the minimum possible sidelobe level in concentric ring arrays [9]. Optimization of the ring radii has been attempted for beam scanning at boresight with the lowest peak SLL [10], but this was done with respect to suppressing the SLL only for the given plane.

To the best of our knowledge, optimization of the concentric ring array geometry for 3D beam scanning with minimum peak SLL has not been addressed. In this paper, optimizing the element placement in a concentric ring array for 3D beam scanning for minimizing sidelobe levels is proposed. In order to achieve 3D beam scanning with the lowest peak SLL, both the radius and the interelement spacing of each ring are optimized by a differential evolution algorithm. Through the optimization, the peak SLL of the optimal concentric ring array is about $6 \mathrm{~dB}$ lower than that of the uniform concentric ring array, but the directivity is reduced by only $0.3 \mathrm{~dB}$. 


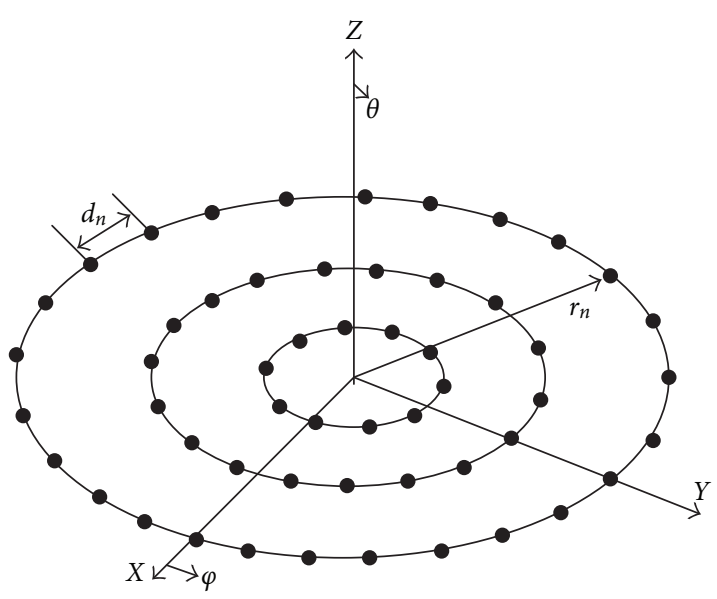

FIgURE 1: Geometry of a concentric circular ring array.

\section{Formulation of the Problem}

Here, consider a concentric ring array having $N$ rings and $M_{n}$ elements in ring $n$. The maximum radii of concentric ring arrays is $R$. The geometry of the concentric ring array of point sources is shown in Figure 1, where $\varphi$ denotes the azimuth angle and $\theta$ the elevation angle with respect to the $Z$-axis. The array factor of the concentric ring array with the uniform excitation amplitude of each ring is given as follows:

$$
\begin{array}{r}
A F(\theta, \varphi)=\sum_{n=1}^{N} \sum_{m=1}^{M_{n}} \exp \left[\left(j k r_{n} \sin \theta \cos \left(\varphi_{m n}-\varphi\right)\right.\right. \\
\left.\left.-\sin \theta_{0} \cos \left(\varphi_{m n}-\varphi_{0}\right)\right)\right],
\end{array}
$$

where $r_{n}$ is the radii distance to the $n$th ring from the center of the array; $k=2 \pi / \lambda$ is the free-space propagation constant, and $\lambda$ is the operating wavelength. The elements in each ring are also assumed to be uniformly distributed around the circular ring, that is, $\varphi_{m n}=2 \pi(m-1) / M_{n} ;\left(\theta_{0}, \varphi_{0}\right)$ is the desired steering angle; $u=\sin \theta \cos \varphi, v=\sin \theta \sin \varphi$. by

The number of equally spaced elements in ring $n$ is given

$$
M_{n}=\left\lfloor\frac{2 \pi r_{n}}{d_{n}}\right\rfloor,
$$

since the number of elements must be an integer, the value in (2) must be rounded up or down. To keep $d \geq \lambda / 2$ and allow sufficient element spacing, the digits to the right of the decimal point are dropped.

For optimization design of the concentric ring array geometry, both the radius of each ring $\mathbf{R}=\left(r_{1}, r_{2}, \ldots, r_{n}\right)^{T}$ and the interelement spacing in each ring $\mathbf{D}=\left(d_{1}, d_{2}, \ldots, d_{n}\right)^{T}$ can be chosen as the optimal variables. The major advantage of the use of optimization of both the ring radii and interelement spacings is that it can enhance computational efficiency by greatly reducing the dimensionality of the space in which any optimization procedure employed in the design process is carried out. In order to alleviate possible mutual coupling effects, the rings are assumed to be separated by a minimum distance of $0.5 \lambda$ and $r_{1} \geq 0.5 \lambda$, and the interelement spacing in each ring is also at least $0.5 \lambda$.

To achieve the lowest peak SLL within the desired steering angle $\left(\theta_{0}, \varphi_{0}\right)$ in $3 \mathrm{D}$ space, the optimization problem is formulated as the following problem:

$$
f(\mathbf{R}, \mathbf{D})=\max _{(\theta, \varphi) \in S}\left|\frac{A F(\theta, \varphi)}{A F_{\max }}\right|,
$$

where $S$ denotes the sidelobe region; $A F_{\max }$ is the peak value of main beam.

\section{Numerical Examples}

In this paper, a $\mathrm{DE} / \mathrm{rand} / 1$ version of $\mathrm{DE}$ is employed to optimize concentric ring arrays. Of course, other global optimization algorithms could also be successfully applied. More details about the DE algorithms refer to [11]. The DE used a scaling factor $F=0.5$, a crossover rate $\mathrm{CR}=0.9$, population size $N P=60$, and the maximum number of generations $G_{\max }=1000$.

In this section, a concentric ring array having $N$ rings is optimized by the DE algorithm. Without loss of generality, the number of rings is $N=6, R=5.0 \lambda$ is considered as the maximum radii of the concentric ring array, and $\left(\theta_{0}, \varphi_{0}\right)=$ $\left(45^{\circ}, 45^{\circ}\right)$ is the desired steering angle. For the array, the average ring spacing is $0.8333 \lambda$. Placing 6 rings at the periodic intervals and the spacing between elements with an approximately constant $(\simeq \lambda / 2)$ for all rings will create relatively high SLL and limit the usefulness of the array. The number of array elements on each ring is then found from (2) as $\left[M_{1}, M_{2}, M_{3}, M_{4}, M_{5}, M_{6}\right]=[10,20,31,41,52,62]$, from the innermost to the outermost ring. The total number of array elements is 216 . Figure 2 shows the patterns of the worst normalized SLL throughout the scanning space. Here the highest SLL is $-16.15 \mathrm{~dB}$, and the directivity is $26.46 \mathrm{~dB}$. The radiation pattern in $\varphi=45^{\circ}$ plane of the array is plotted in Figure 3.

Optimizing D: to reduce the peak SLL, we try to optimize the element spacings of all the rings $\mathbf{D}=\left(d_{1}, d_{2}, \ldots, d_{6}\right)$. $d_{n}$ is limited between $0.5 \lambda$ and $\lambda$. The radii of ring $\mathbf{R}=\left(r_{1}, r_{2}, \ldots, r_{6}\right)$ is placed for $(0.8333 \lambda, 1.6667 \lambda, 2.5000 \lambda$, $4.1667 \lambda, 5.0000 \lambda)$. The DE is employed to optimize element spacings of all the rings. The optimal element spacings of the 6 rings by the $\mathrm{DE}$ algorithm are $(0.5432 \lambda, 0.5834 \lambda$, $0.5696 \lambda, 0.6281 \lambda, 0.5210 \lambda, 0.5056 \lambda)$. The number of array elements on each ring is $\left[M_{1}, M_{2}, M_{3}, M_{4}, M_{5}, M_{6}\right]=$ $[9,17,27,33,50,62]$, and the resulting array has 198 elements arranged as shown in Figures 4 and 5 shows the patterns of the worst SLL at the scanning space. Here the highest SLL is $-16.31 \mathrm{~dB}$, and the directivity is $26.07 \mathrm{~dB}$. The improvement is very small. The radiation pattern in $\varphi=45^{\circ}$ plane of the array is plotted in Figure 6.

Optimizingboth $\mathbf{D}$ and R: Optimizing the element spacings of all the rings for reducing the peak SLL is not obvious, so we can attempt to optimize both the radii and the element spacings of all the rings. For this array, the maximum radius of the ring is $5.0 \lambda$, that is, $r_{6}=R=$ 5.0 $\lambda$. The DE is used to determine how to combine the 


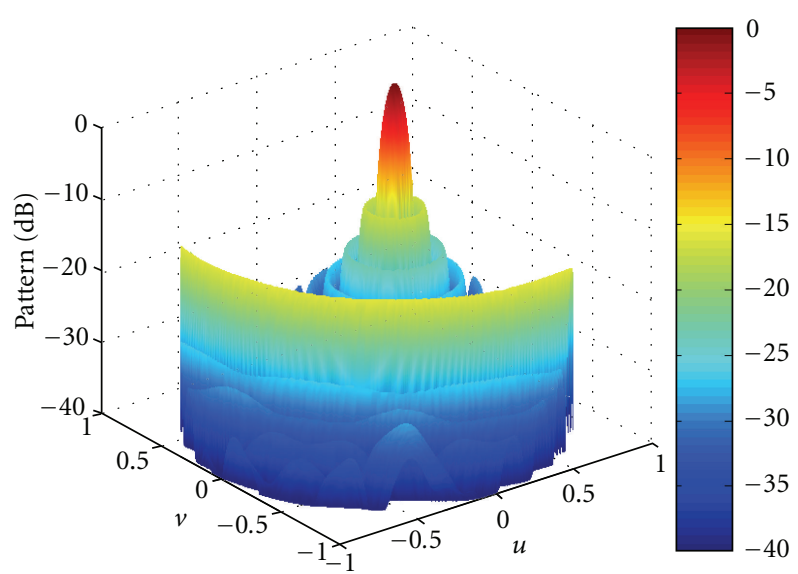

(a)

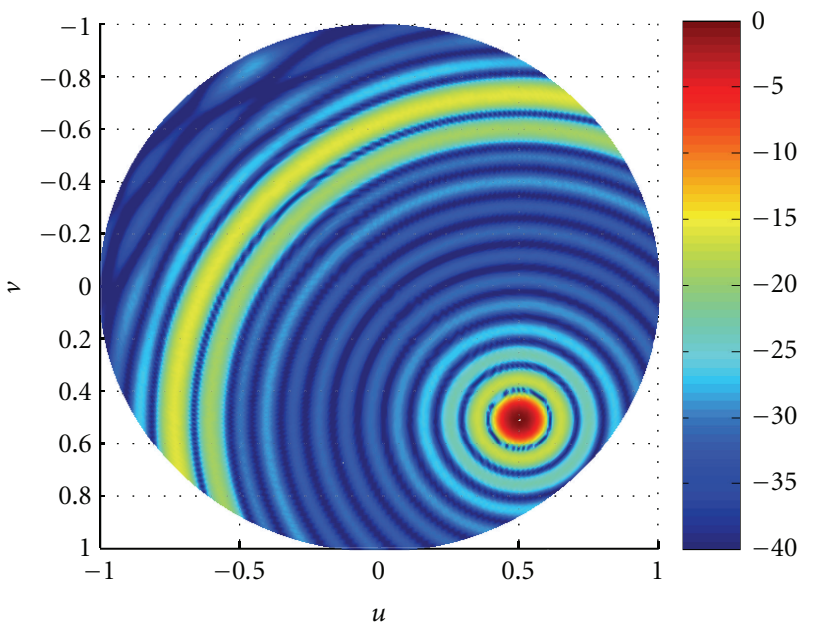

(b)

FIgURE 2: 3D radiation pattern of the uniform concentric circular ring array steered to $\left(45^{\circ}, 45^{\circ}\right)$. (a) $3 \mathrm{D}$ view, (b) Top view.

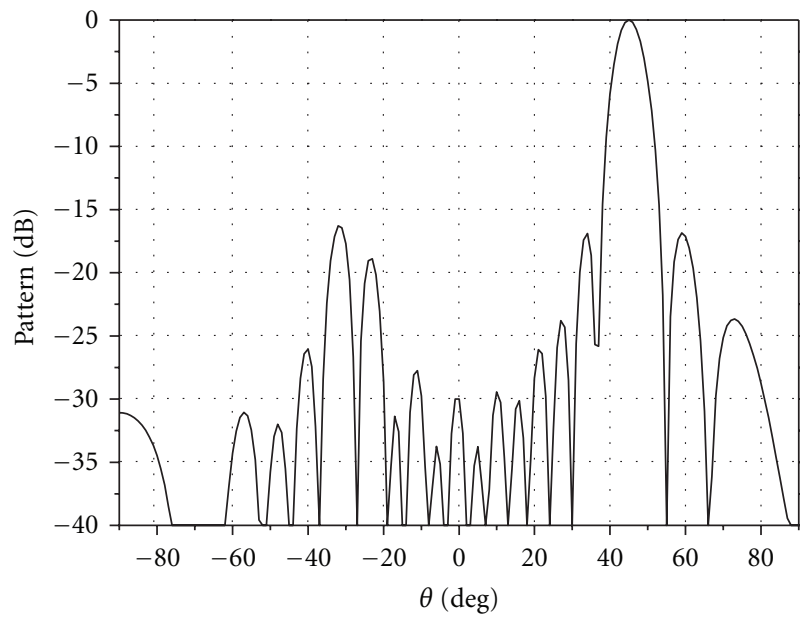

FIGURE 3: Radiation pattern in the $\varphi=45^{\circ}$ plane of the uniform concentric circular ring array.

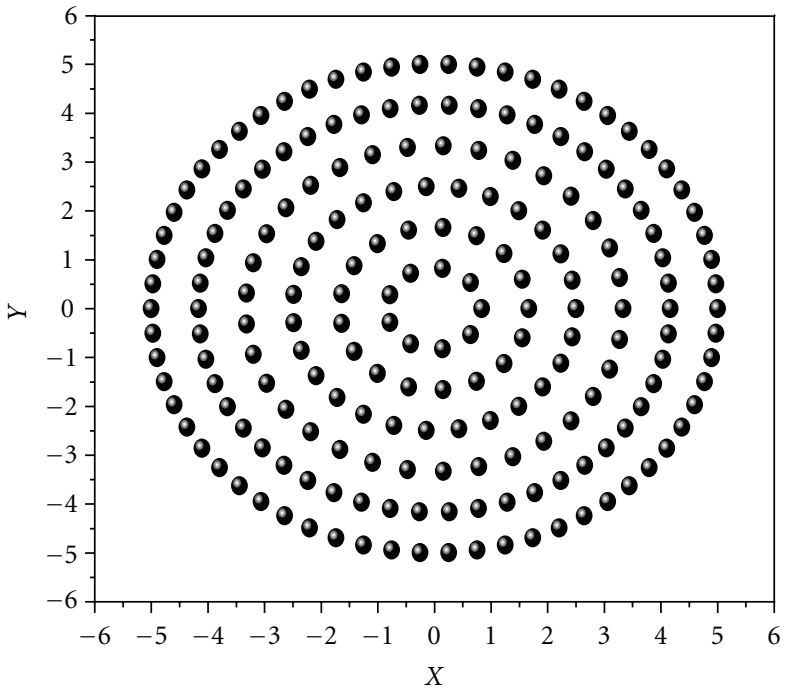

FIgURE 4: The array geometry with only optimizing D.

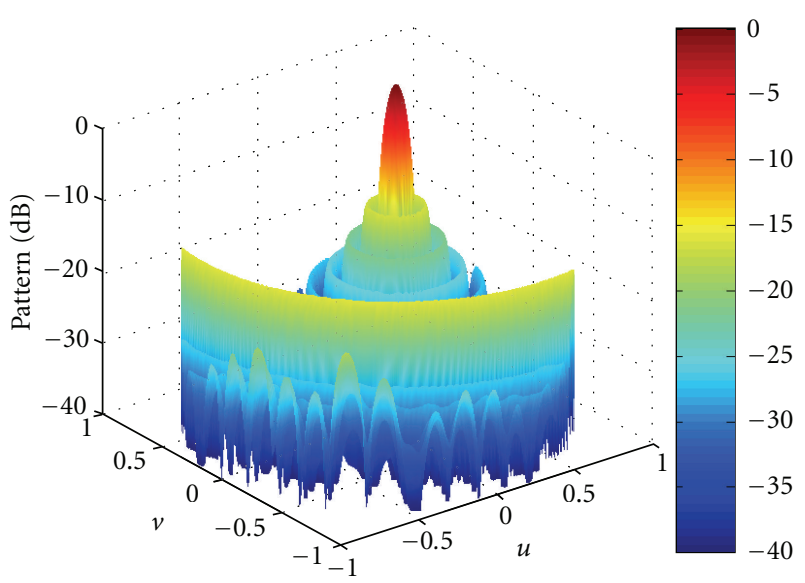

(a)

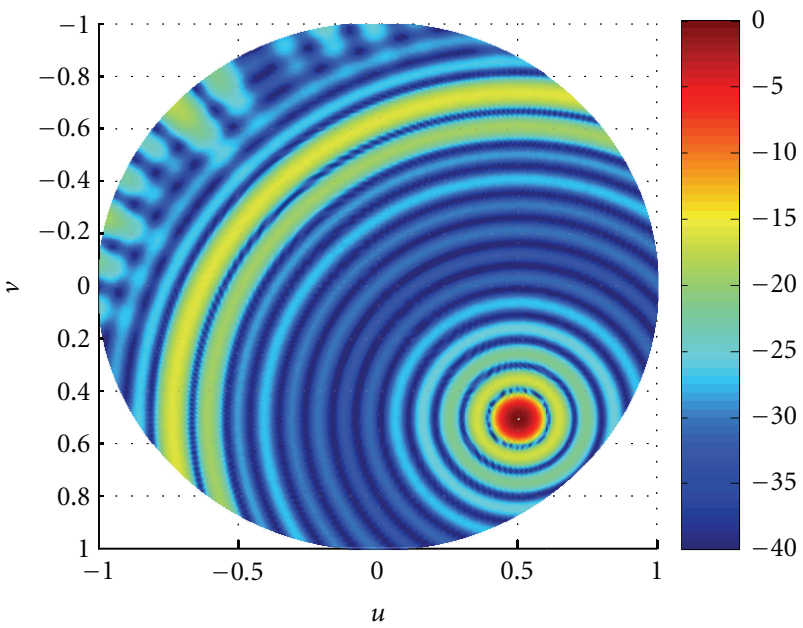

(b)

FIGURE 5: 3D radiation pattern of the array with only optimizing D steered to $\left(45^{\circ}, 45^{\circ}\right)$. (a) $3 \mathrm{D}$ view, (b) Top view. 


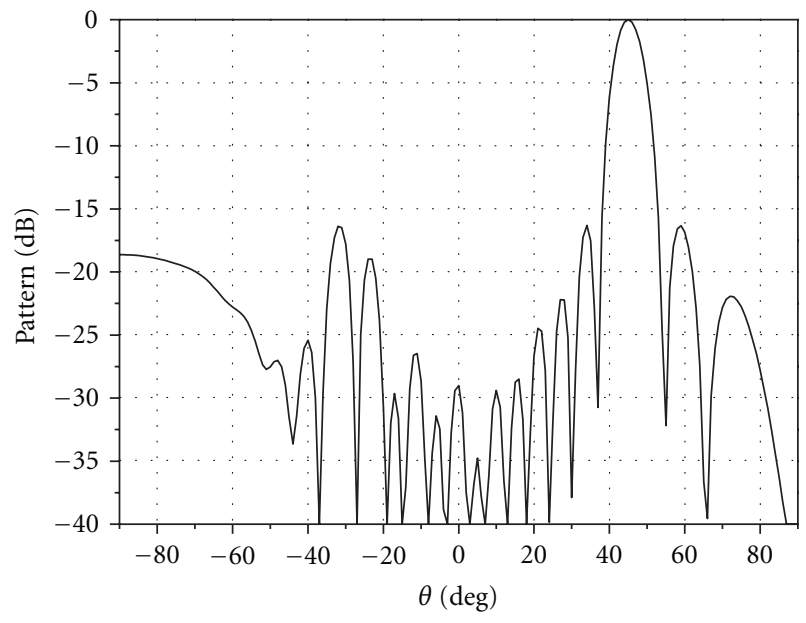

FIGURE 6: Radiation pattern in the $\varphi=45^{\circ}$ plane of the array with only optimizing $\mathbf{D}$.

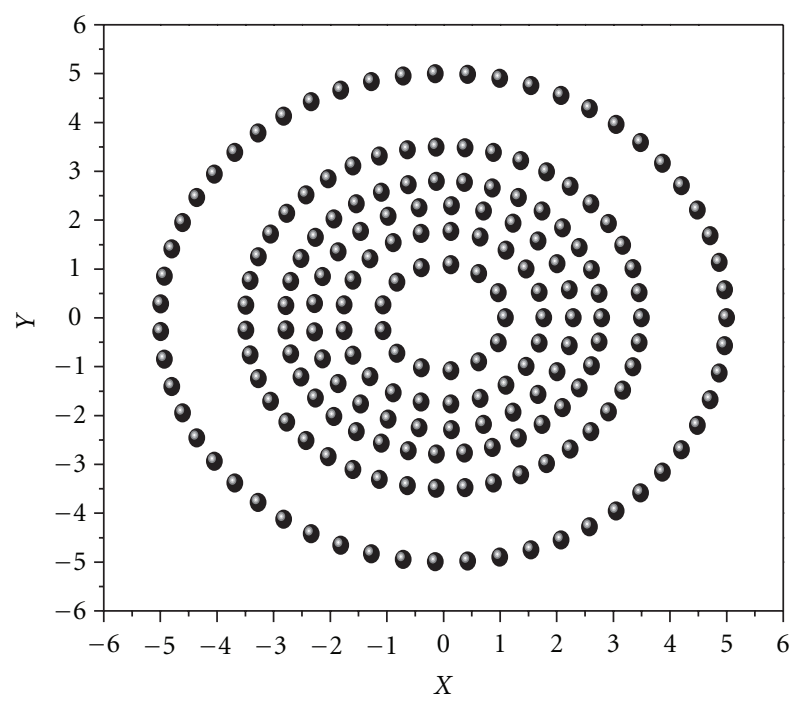

FIgURE 7: The array geometry with optimizing both $\mathbf{D}$ and $\mathbf{R}$.

radii and the element spacings of all the rings to achieve the lowest SLL. In this optimization process, the solution may be infeasible, that is, it is not satisfied the constraints $\left(r_{n}-r_{n-1} \geq 0.5 \lambda, 2 \leq n \leq 6\right.$, and $\left.r_{6}=R=5.0 \lambda\right)$. To avoid this drawback, the constraint-handling technique in [12] is adopted in the DE algorithm. The final optimized results are shown in the following. The optimal radii of the 6 rings are $(1.0964 \lambda, 1.7713 \lambda, 2.2939 \lambda, 2.7939 \lambda, 3.4964 \lambda$, $5.0000 \lambda)$, and the optimal element spacings of the 6 rings are $(0.5251 \lambda, 0.5176 \lambda, 0.5706 \lambda, 0.5000 \lambda, 0.5043 \lambda, 0.5664 \lambda)$. The optimal array has only 192 elements, and the number of elements in each ring is $\left[M_{1}, M_{2}, M_{3}, M_{4}, M_{5}, M_{6}\right]=$ $[13,21,25,35,43,55]$. The optimal geometry of the array is shown in Figure 7. The corresponding patterns are plotted in Figures 8 and 9. The directivity is $26.16 \mathrm{~dB}$ and the peak SLL of the optimal array is $-22.05 \mathrm{~dB}$.

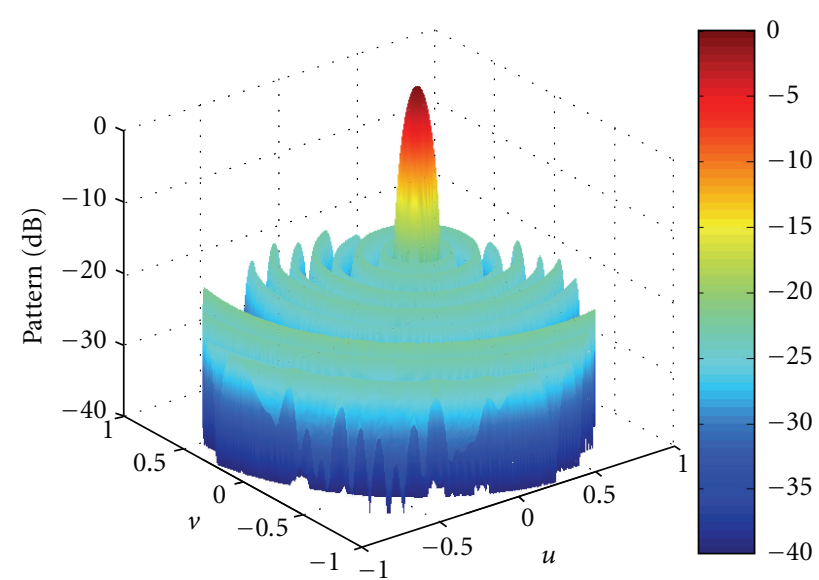

(a)

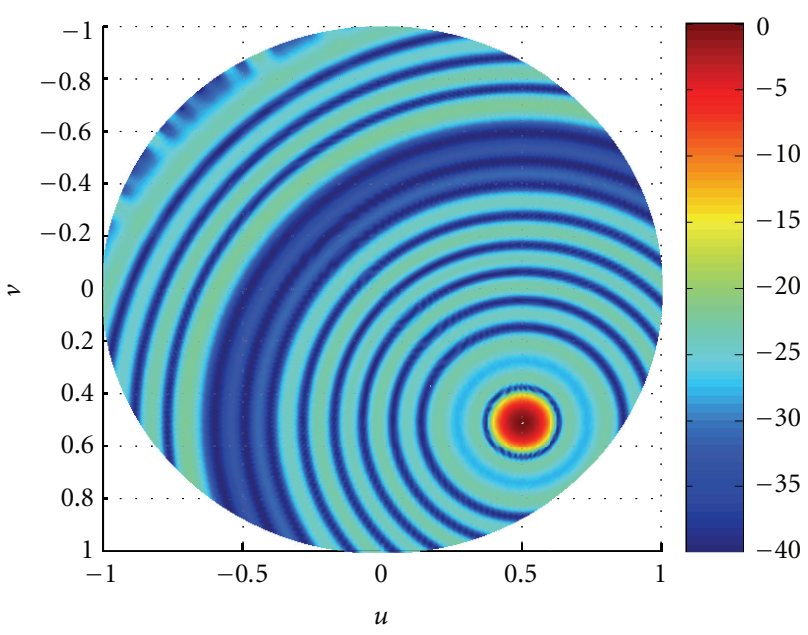

(b)

FigURE 8: 3D radiation pattern of the array with optimizing both $\mathbf{D}$ and $\mathbf{R}$ steered to $\left(45^{\circ}, 45^{\circ}\right)$. (a) 3D view, (b) Top view.

TABLE 1: The results of the cases presented.

\begin{tabular}{lccc}
\hline Array & Peak sidelobe level & Directivity & Number of elements \\
\hline Uniform & $-16.15 \mathrm{~dB}$ & $26.46 \mathrm{~dB}$ & 216 \\
Opt. D & $-16.31 \mathrm{~dB}$ & $26.07 \mathrm{~dB}$ & 198 \\
Opt. R \& D & $-22.05 \mathrm{~dB}$ & $26.16 \mathrm{~dB}$ & 192 \\
\hline
\end{tabular}

The performance parameters of the arrays in the presented cases are listed in Table 1 . As can be seen from Table 1, the lowest sidelobe level of the concentric ring array is obtained by optimizing both the ring radii and the inter-element spacing in each ring. This array has the fewest number of elements so far at 192 or $88.9 \%$ of the uniform concentric circular array. Directivity analysis shows that the methodology not only provides an effective way to regulate the radiation patterns but also is comparable in efficiency to standard array geometries. The directivity of the uniform array, the array with only optimizing $\mathbf{D}$, and the array with optimizing both $\mathbf{D}$ and $\mathbf{R}$ were found to be $26.46 \mathrm{~dB}, 26.07 \mathrm{~dB}$, and $26.16 \mathrm{~dB}$, respectively. Compared 


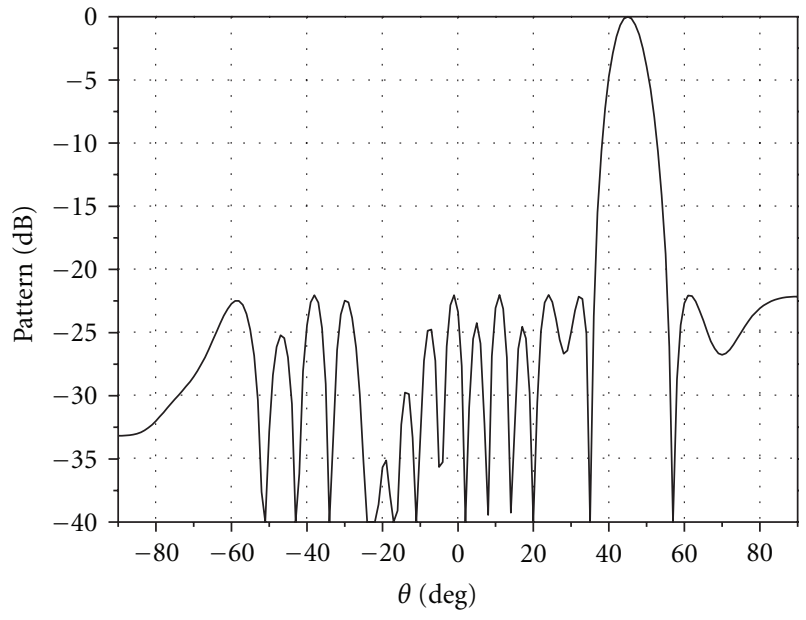

FIGURE 9: Radiation pattern in the $\varphi=45^{\circ}$ plane of the array with optimizing both $\mathbf{D}$ and $\mathbf{R}$.

with the uniform design, the highest SLL of the optimal array is reduced by about $6 \mathrm{~dB}$, but the directivity is reduced by only $0.3 \mathrm{~dB}$. Among these arrays, the uniform array has the highest directivity, but the SLL is also the highest; the optimal array (optimizing both the ring radii and the interelement spacing in each ring) has the lowest SLL and moderate directivity.

\section{Conclusion}

In this paper, optimization of the concentric ring array geometry for 3D beam scanning to minimize sidelobe levels is proposed. Both the ring radius and the number of elements in each ring are optimized to achieve 3D beam scanning with the lowest peak SLL. The optimization problem is solved via the differential evolution algorithm. Through the optimization, the peak SLL of the optimal concentric ring array is about $6 \mathrm{~dB}$ lower than that of the uniform concentric ring array. It is found that array geometry has a significant effect on the performance of the concentric ring arrays.

\section{Acknowledgment}

This work was supported by the Fundamental Research Funds for the Central Universities (K50511020007).

\section{References}

[1] C. A. Balanis, Antenna Theory: Analysis and Design, John Wiley \& Sons, New York, NY, USA, 2nd edition, 1997.

[2] M. Shihab, Y. Najjar, N. Dib, and M. Khodier, "Design of non-uniform circular antenna arrays using particle swarm optimization," Journal of Electrical Engineering, vol. 59, no. 4, pp. 216-220, 2008.

[3] M. A. Panduro, C. A. Brizuela, L. I. Balderas, and D. A. Acosta, "A comparison of genetic algorithms, particle swarm optimization and the differential evolution method for the design of scannable circular antenna arrays," Progress In Electromagnetics Research B, no. 13, pp. 171-186, 2009.
[4] Y. Li, K. C. Ho, and C. Kwan, "3-D array pattern synthesis with frequency invariant property for concentric ring array," IEEE Transactions on Signal Processing, vol. 54, no. 2, pp. 780-784, 2006.

[5] B. P. Kumar and G. R. Branner, "Generalized analytical technique for the synthesis of unequally spaced arrays with linear, planar, cylindrical or spherical geometry," IEEE Transactions on Antennas and Propagation, vol. 53, no. 2, pp. 621-634, 2005.

[6] H. Ei-Kamchouchi, "Ultra-low-sidelobe-level concentricring-array pattern synthesis using Bessel neural networks," IEEE Antennas and Propagation Magazine, vol. 52, no. 4, pp. 102-105, 2010.

[7] R. L. Haupt, "Optimized element spacing for low sidelobe concentric ring arrays," IEEE Transactions on Antennas and Propagation, vol. 56, no. 1, pp. 266-268, 2008.

[8] N. Pathak, G. K. Mahanti, S. K. Singh, J. K. Mishra, and A. Chakraborty, "Synthesis of thinned planar circular array antennas using modified particle swarm optimization," Progress in Electromagnetics Research Letters, vol. 12, pp. 87-97, 2009.

[9] F. Li, Y.-C. Jiao, L.-S. Ren, Y.-Y. Chen, and L. Zhang, "Pattern synthesis of concentric ring array antennas by differential evolution algorithm," Journal of Electromagnetic Waves and Applications, vol. 25, no. 2, pp. 421-430, 2011.

[10] G. Holtrup, A. Marguinaud, and J. Citerne, "Synthesis of electronically steerable antenna arrays with elements on concentric rings with reduced sidelobes," in Proceedings of the IEEE Antennas and Propagation Society International Symposium, pp. 800-803, July 2001.

[11] R. Storn and K. Price, "Differential evolution-a simple and efficient heuristic for global optimization over continuous spaces," Journal of Global Optimization, vol. 11, no. 4, pp. 341359, 1997.

[12] K. Chen, Z. He, and C. Han, "A modified real GA for the sparse linear array synthesis with multiple constraints," IEEE Transactions on Antennas and Propagation, vol. 54, no. 7, pp. 2169-2173, 2006. 

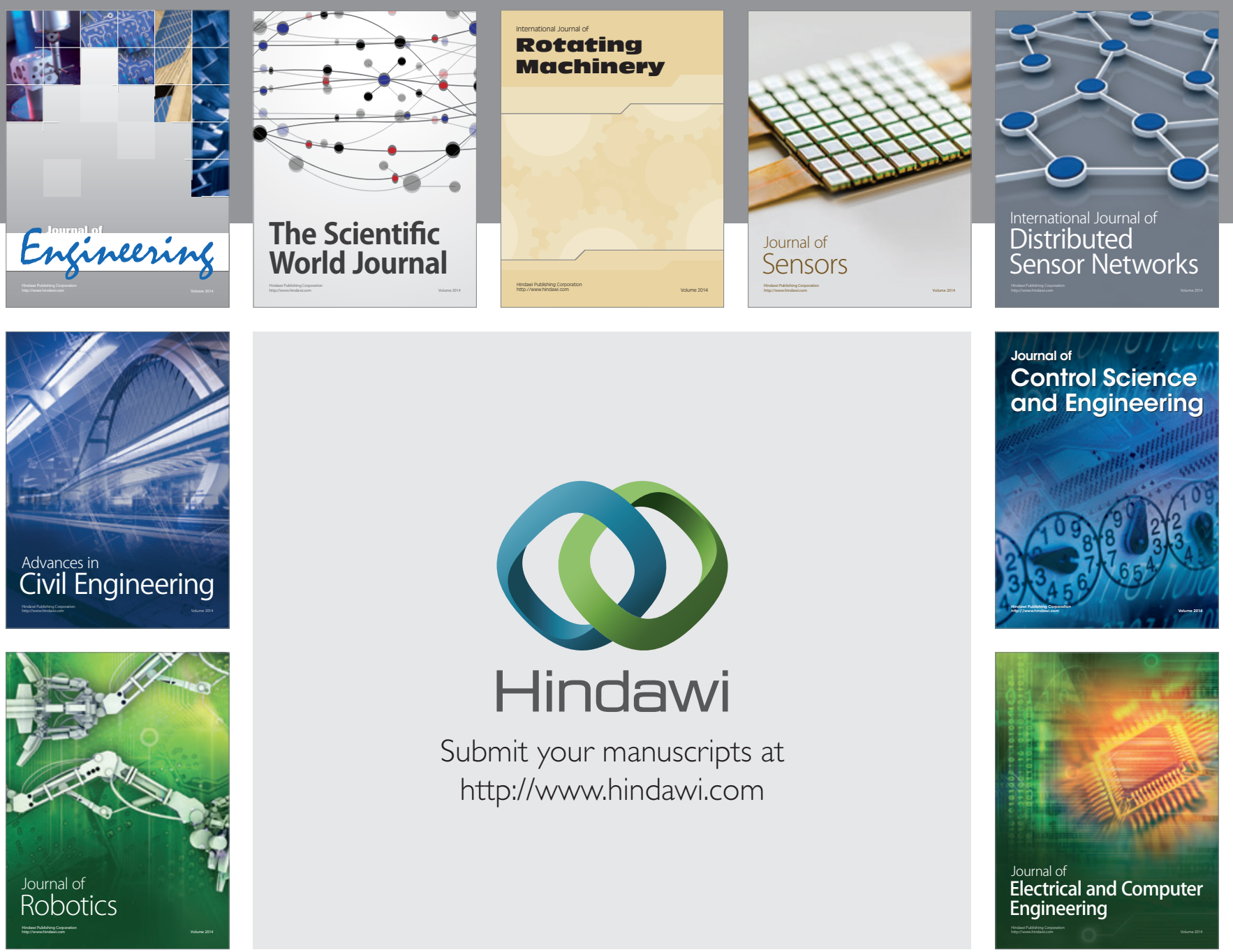

Submit your manuscripts at

http://www.hindawi.com
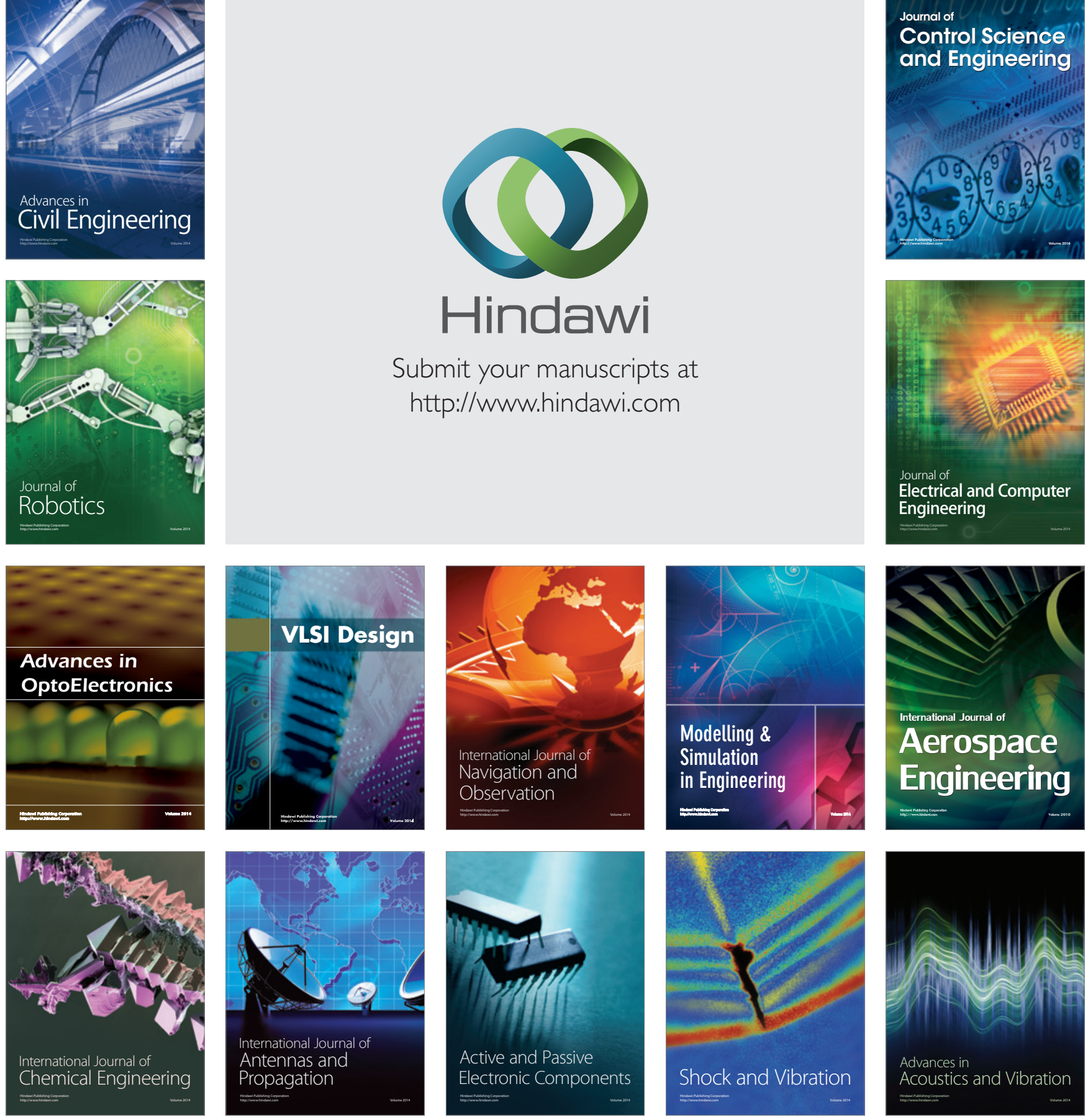